\title{
Clinical Usefulness of Amisulpride Add-on Therapy in Schizophrenia Patients without Treatment Response to Second-generation Antipsychotics
}

\author{
Seung-Gul Kang ${ }^{1}$, Seo-Eun Cho ${ }^{1}$, Kyoung-Sae $\mathrm{Na}^{1}$, Chi-Un Pae ${ }^{2,3}$, Seong-Jin Cho ${ }^{1}$ \\ 'Department of Psychiatry, Gil Medical Center, Gachon University College of Medicine, Incheon, ${ }^{2}$ Department of Psychiatry, Bucheon St. Mary's \\ Hospital, ${ }^{3}$ Cell Death Disease Research Center, College of Medicine, The Catholic University of Korea, Bucheon, Korea
}

\begin{abstract}
Objective: The response to antipsychotics in patients with schizophrenia is still unsatisfactory. Therefore, augmentation with other antipsychotics is common in clinical situations. The purpose of this study was to evaluate the improvement of psychiatric symptoms and side effects after amisulpride add-on therapy.

Methods: Forty patients with schizophrenia or schizoaffective disorder without treatment response to second-generation antipsychotics were included in this study. Psychotic symptoms were evaluated using the Positive and Negative Syndrome Scale (PANSS) and the Korean version of Calgary Depression Scale for Schizophrenia (CDSS) at baseline, 4 weeks, and 8 weeks after the addition of amisulpride.

Results: Among the 29 subjects who completed the 8-week study, 34.5\% were responders according to PANSS total score. At week 8 , the mean positive $(p<0.001)$, negative $(p<0.001)$, general $(p<0.001)$, and total $(p<0.001)$ PANSS scores and CDSS scores $(p=0.002)$ showed significant improvement compared to baseline. There was no increase in extrapyramidal side effects according to Simpson Angus Scale $(p=0.379)$ and Barnes Akathisia Rating Scale $(p=0.070)$ and no weight gain $(p=0.308)$ after the add-on treatment.

Conclusion: The addition of amisulpride for schizophrenia patients without therapeutic response to second-generation antipsychotics is considered an effective and safe treatment. This study's results suggested that augmentation of second-generation antipsychotics with amisulpride could be a useful option for patients with schizophrenia unresponsive to second-generation antipsychotics. Further studies investigating the efficacy of amisulpride add-on therapy using placebo control are necessary to confirm these results.
\end{abstract}

KEY WORDS: Antipsychotic agents; Amisulpride; Drug augmentation; Schizophrenia; Treatment efficacy.

\section{INTRODUCTION}

Antipsychotics are mainstays in the treatment of schizophrenia and other psychotic disorders. Although many antipsychotics have been developed and the side effects of antipsychotics have decreased considerably, the treatment responses of patients with schizophrenia remain unsatisfactory. Approximately $20-30 \%$ of the patients with schizophrenia do not show treatment response to an-

Received: April 7, 2020 / Revised: July 10, 2020

Accepted: July 13, 2020

Address for correspondence: Seong-Jin Cho

Department of Psychiatry, Gil Medical Center, Gachon University College of Medicine, 21 Namdong-daero 774beon-gil, Namdong-gu, Incheon 21565, Korea

E-mail: sjcho@gilhospital.com

ORCID: https://orcid.org/0000-0002-8814-5807 tipsychotics [1]. Treatment resistance of schizophrenia might result in significant sequelae including functional disability at work and in education, social withdrawal, and even suicide. Clozapine is the only effective medication for treatment-resistant schizophrenia [2]. However, it has significant side effects such as agranulocytosis, seizure, sialorrhea, weight gain, and sedation [2].

Therefore, add-on therapies with antipsychotics have been attempted frequently in patients with refractory schizophrenia in clinical situations and many studies have been conducted on this topic [3-5]. Acute patients who did not respond to initial and second antipsychotics showed effective and relatively tolerable response to a third antipsychotic administered as an augmentation therapy [5]. A systematic review of randomized controlled tri-

(c) This is an Open-Access article distributed under the terms of the Creative Commons Attribution Non-Commercial License (http://creativecommons.org/licenses/by-nc/4.0) which permits unrestricted non-commercial use, distribution, and reproduction in any medium, provided the original work is properly cited. 
als (RCTs) regarding augmentation with aripiprazole in patients with schizophrenia resistant to clozapine suggested trends of aripiprazole augmentation benefits on overall positive and negative symptoms. However, the improvement was not significant [3]. Another meta-analysis on the same topic showed significant improvement in symptoms when the augmentation treatment lasted for more than 10 weeks. However, there was a lack of improvement when the treatment duration was less than 10 weeks [4]. A meta-analysis comparing antipsychotic augmentation therapy and monotherapy showed that augmentation therapy lacked double-blind/high-quality evidence for efficacy, except the reported negative symptom reduction with aripiprazole augmentation [6]. Generally, research results on augmentation therapy have been inconsistent with respect to the combination of augmentation drugs and research design.

Amisulpride is an effective benzamide antipsychotic with high selectivity for $\mathrm{D}_{2}$ and $\mathrm{D}_{3}$ dopamine receptors. It has been reported to be as effective as haloperidol $[7,8]$ and olanzapine [9] in treating schizophrenia. In a study comparing amisulpride and haloperidol, amisulpride had a comparable effect on positive symptoms and a superior effect on negative symptoms when compared with haloperidol [8]. In a meta-analysis by Leucht et al. [10], amisulpride showed robust efficacy and better tolerability with less discontinuation due to side effects when compared with a placebo. Several studies have reported the efficacy of amisulpride augmentation therapy in patients with schizophrenia who showed partial response to antipsychotics [11-14]. These studies generally reported that augmentation with amisulpride was a useful and safe treatment. However, the number of studies was insufficient to generalize these results and additional studies were recommended since there were no consistent results regarding the side effects $[11,14]$. Moreover, no research has been done on this subject in Korea.

The present study was designed to determine whether amisulpride augmentation therapy had good efficacy and tolerability in Korean patients with schizophrenia who showed insufficient treatment response to second-generation antipsychotics (SGAs).

\section{METHODS}

\section{Subjects}

Board-certified psychiatrists interviewed the patients using the Diagnostic and Statistical Manual of Mental Disorders, fifth edition (DSM-5) criteria [15]. The inclusion criteria were as follows: 1) men and women aged 18 65 years who were diagnosed with schizophrenia or schizoaffective disorder according to the DSM-5 diagnostic criteria, 2) insufficient treatment response to SGAs for more than 2 months (defined as a score of 4 or higher for both hallucinations and delusions on the Positive and Negative Syndrome Scale (PANSS) [16]), and 3) patients who fully understood the study and provided written consent. The exclusion criteria were as follows: 1) serious medical or neurological diseases other than schizophrenia, 2) subjects who had taken clozapine previously, 3) pregnant or breastfeeding females, and 4) treatment with long-acting injectable antipsychotics during the previous month. All the participants provided written informed consent and the Institutional Review Board of Gil Medical Center approved this study protocols before the commencement of the study (no. GBIRB2014-64). We followed the relevant guidelines and regulations for all experiments throughout the study period.

\section{Amisulpride Treatment and Clinical Assessments}

Patients were administered $400 \mathrm{mg}$ of amisulpride orally while maintaining the previous antipsychotic drugs. Generally, if there is a history of extrapyramidal side effects (EPS) or a concern about such side effects, a $200 \mathrm{mg}$ dose may be administered and the increase of dosage to $400 \mathrm{mg}$ should be considered after 2 weeks. In the present study, amisulpride was administered as a fixed dose for 4 weeks. After 4 weeks, it could be changed to a flexible dose depending on the symptoms and the side effects of the drug.

Benztropine was allowed to be administered when EPS occurred. Same doses of previous antipsychotic drugs, mood stabilizers, sleeping pills, anticholinergic drugs, antidepressants, and anti-anxiety drugs taken during previous 2 weeks were maintained during the study period. No other antipsychotic drugs were allowed during the entire study period.

Psychiatric symptoms and side effects of the antipsychotics were evaluated at baseline, 4 weeks, and 8 
weeks. Psychiatric symptoms and their intensity in each participant were evaluated using PANSS [16], Clinical Global Impression Scale (CGI-S) [17], Clinical Global Impression Clinical Benefit Scale (CGI-CB), and the Korean version of Calgary Depression Rating Scale for Schizophrenia (CDSS) $[18,19]$. Side effects of antipsychotics were evaluated using Barnes Akathisia Rating Scale (BARS) [20] and Simpson Angus Scale (SAS) [21]. Each patient was interviewed and evaluated by the same psychiatrist throughout the study period.

\section{Outcome Measure and Statistical Analysis}

The primary goal of the present study was to observe the improvement of psychiatric symptoms according to PANSS scores at 4 weeks and at 8 weeks after the addition of amisulpride to previous SGAs and to investigate the percentage of responders to augmentation.

The secondary goal of the study was to assess the degree of global improvement based on CGI-S scores at 4 and 8 weeks. Depression severity was assessed using CDSS at 4 and 8 weeks. Evaluation of EPS and weight gain was performed using body mass index (BMI), BARS, and SAS.

Treatment response was defined as a decrease in the PANSS total score of more than $50 \%$ of the baseline score minus 30 (i.e., minimum possible PANSS total score), as described previously [22].

if $x$ was $>50$, subjects were considered to show a response.

$x=\frac{\begin{array}{c}\text { (baseline PANSS score minus } \\ \text { endpoint PANSS score }) \times 100\end{array}}{(\text { baseline PANSS score minus 30) }}$

At the start of the clinical study, demographic characteristics (age and sex) and clinical characteristics of all the participants were assessed. The quantitative data were presented as mean \pm standard deviation and the qualitative data were presented as frequency and percentage.

Descriptive statistics, frequency analysis, chi-squared test, independent $t$ test, and paired $t$ test were used for the statistical analyses. All analyses were performed using SPSS Statistics 25.0 (IBM Corp., Armonk, NY, USA). The level of statistical significance was set at $p<0.05$.

\section{RESULTS}

\section{Characteristics of the Participants}

Among the 40 subjects included in the study, 29 subjects completed the treatment course and the follow-up evaluation for 8 weeks (Table 1). The mean age of the participants was $45.5 \pm 14.0$ years, the mean duration of schizophrenia was $13.6 \pm 9.9$ years, and the mean number of antipsychotic drugs used were $1.2 \pm 0.4$. The average PANSS score of the subjects was $81.9 \pm 14.3(81.7 \pm$ 12.5 in completers) and the mean CGI-S score was $4.4 \pm$ 0.9 (4.5 \pm 0.9 in completers). Thus, all the participants were in moderately ill to markedly ill state.

Subjects were those who had been taking one or two antipsychotics. The number of subjects who took and dosage of each antipsychotic were as follows: paliperidone $(n=18$, mean dosage $=10.2 \pm 3.9 \mathrm{mg})$, olanzapine $(n=5$, mean dosage $=13.5 \pm 6.0 \mathrm{mg})$, risperidone $(n=5$, mean dosage $=6.2 \pm 3.5 \mathrm{mg}$ ), blonanserin $(\mathrm{n}=8$, mean dosage $=13.5 \pm 6.7 \mathrm{mg})$, aripiprazole $(n=5$, mean dosage $=$ $23.0 \pm 11.0 \mathrm{mg})$, quetiapine $(\mathrm{n}=6$, mean dose $=79.2 \pm$ $69.7 \mathrm{mg}$ ), and chlorpromazine ( $\mathrm{n}=1$, dosage $=100 \mathrm{mg}$ ). The average duration of antipsychotics use was $12.5 \pm$ 14.0 months. Eight people had taken two antipsychotics, the majority of whom $(n=6)$ were using quetiapine or chlorpromazine for the purpose of improving sleep. There was no statistically significant relationship between the number of antipsychotic drugs used and the response at 8 weeks $\left(\chi^{2}=1.06, p=0.303\right)$.

Table 1. Demographic and clinical characteristics of participants at baseline

\begin{tabular}{lcc}
\hline \multicolumn{1}{c}{ Variable } & $\begin{array}{c}\text { All patients } \\
(\mathrm{n}=40)\end{array}$ & $\begin{array}{c}\text { Completers } \\
(\mathrm{n}=29)\end{array}$ \\
\hline Age $(\mathrm{yr})$ & $45.5 \pm 14.0$ & $45.6 \pm 13.6$ \\
Sex, female & $21(52.5)$ & $14(48.3)$ \\
Duration of schizophrenia $(\mathrm{yr})$ & $13.6 \pm 9.9$ & $14.5 \pm 10.3$ \\
Body mass index & $25.5 \pm 4.5$ & $25.0 \pm 3.9$ \\
Number of prior antipsychotics & $1.2 \pm 0.4$ & $1.2 \pm 0.4$ \\
Starting dose of amisulpride & $330.0 \pm 96.6$ & $331.0 \pm 96.7$ \\
PANSS total score & $81.9 \pm 14.3$ & $81.7 \pm 12.5$ \\
CGl-S & $4.4 \pm 0.9$ & $4.5 \pm 0.9$ \\
SAS & $0.9 \pm 2.2$ & $1.3 \pm 2.5$ \\
BARS & $1.0 \pm 1.3$ & $0.9 \pm 1.4$ \\
\hline
\end{tabular}

Data are presented as mean \pm standard deviation or number (percentage). PANSS, Positive And Negative Syndrome Scale; CGI-S, Clinical Global Impression Severity Scale; SAS, Simpson Angus Scale; BARS, Barnes Akathisia Rating Scale. 


\section{Efficacy Evaluation}

Among the 29 subjects who completed this study, $34.5 \%$ showed symptom improvement of $50 \%$ or more according to the PANSS total score (Table 2). Average starting dose of amisulpride was $331.0 \pm 96.7 \mathrm{mg}$ in patients who completed the study (Table 3). After 8 weeks, $30 \%$ of the patients in the $200 \mathrm{mg}$ starting dose group and $36.7 \%$ of the patients in the $400 \mathrm{mg}$ starting dose group responded to the treatment. There was no significant difference in the number of responders between the two groups $\left(\chi^{2}=0.14, p=0.713\right.$; Table 2$)$.

Pre-treatment and post-treatment (after 8 weeks) PANSS, CDSS, CGI-S, and CGI-CB scores were compared in subjects who completed the study. PANSS scores in all domains (positive: $t=-10.92, p<0.001$; negative: $t=$ $-7.63, p<0.001$; general: $t=-7.83, p<0.001$ ) and PANSS total scores $(t=-11.80, p<0.001)$ showed significant changes. CDSS $(t=-3.34, p=0.002), \mathrm{CGI}-\mathrm{S}(t=$ $-8.07, p<0.001)$, and CGI-CB $(t=-7.41, p<0.001)$ scores showed significant improvement (Table 3 ).

There were no statistically significant differences in demographic characteristics (age and sex), duration of illness, and number of antipsychotic drugs between the 200 mg starting dose group and the $400 \mathrm{mg}$ starting dose group (Table 4). The PANSS and CGI-S scores at baseline and the scores after 8 weeks did not show statistically sig-

Table 2. Response rate and premature withdrawal from the study $(n=40)$

\begin{tabular}{|c|c|c|c|c|}
\hline Variable & Total $(n=40)$ & $\begin{array}{l}200 \mathrm{mg} \text { start } \\
\text { group }(\mathrm{n}=14)\end{array}$ & $\begin{array}{l}400 \mathrm{mg} \text { start } \\
\text { group }(\mathrm{n}=26)\end{array}$ & $\begin{array}{c}\text { Comparison between } 200 \mathrm{mg} \\
\text { and } 400 \mathrm{mg} \text { start groups }\end{array}$ \\
\hline $\begin{array}{l}\text { Responders among completers }(n=29) \\
\text { based on PANSS total score }\end{array}$ & $10 / 29(34.5)$ & $3 / 10(30.0)$ & $7 / 19(36.8)$ & $\chi^{2}=0.14, p=0.713$ \\
\hline Total withdrawals & $11(27.5)$ & $4(28.6)$ & $7(26.9)$ & $\chi^{2}=0.01, p=0.911$ \\
\hline Reason for withdrawal & & & & $\chi^{2}=8.27, p=0.082$ \\
\hline Lack of efficacy & $2(5)$ & $2(14.3)$ & $0(0)$ & \\
\hline Adverse reaction & $3(7.5)$ & $2(14.3)$ & $1(3.8)$ & \\
\hline Lost to follow-up & $4(10)$ & $0(0)$ & $4(15.4)$ & \\
\hline Other reasons & $2(5)$ & $0(0)$ & $2(7.7)$ & \\
\hline Time of withdrawal & & & & $\chi^{2}=1.43, p=0.488$ \\
\hline$<4$ weeks & $9(22.5)$ & $4(28.6)$ & $5(19.2)$ & \\
\hline$\geq 4$ weeks and $<8$ weeks & $2(5)$ & $0(0)$ & $2(7.7)$ & \\
\hline
\end{tabular}

Data are presented as number (percentage).

PANSS, Positive And Negative Syndrome Scale.

$\chi^{2}$ : chi-squared test was used for statistical comparisons.

Table 3. Comparison of treatment outcomes and adverse reactions in patients with schizophrenia before and after amisulpride add-on therapy

\begin{tabular}{|c|c|c|c|c|}
\hline Variable & Baseline & 4 weeks & 8 weeks & Comparison (baseline vs. 8 weeks) \\
\hline Dose of amisulpride (mg) & $331.0 \pm 96.7$ & $358.6 \pm 132.3$ & & \\
\hline \multicolumn{5}{|l|}{ PANSS } \\
\hline Total & $81.7 \pm 12.5$ & $67.3 \pm 12.4$ & $60.0 \pm 14.7$ & $t=-11.80, p<0.001$ \\
\hline Positive & $24.0 \pm 4.6$ & $17.8 \pm 4.7$ & $15.3 \pm 4.9$ & $t=-10.92, p<0.001$ \\
\hline Negative & $18.4 \pm 3.7$ & $15.7 \pm 3.0$ & $14.2 \pm 3.4$ & $t=-7.63, p<0.001$ \\
\hline General & $39.7 \pm 8.0$ & $33.7 \pm 6.7$ & $30.8 \pm 7.9$ & $t=-7.83, p<0.001$ \\
\hline CDSS & $3.6 \pm 4.5$ & $1.9 \pm 3.6$ & $1.9 \pm 4.3$ & $t=-3.34, p=0.002$ \\
\hline CGI-S & $4.5 \pm 0.9$ & $3.5 \pm 0.9$ & $3.1 \pm 0.9$ & $t=-8.07, p<0.001$ \\
\hline CGI-CB & $6.8 \pm 2.0$ & $4.3 \pm 1.9$ & $4.0 \pm 1.6$ & $t=-7.41, p<0.001$ \\
\hline \multicolumn{5}{|l|}{ Extrapyramidal side effects } \\
\hline SAS & $1.3 \pm 2.5$ & $1.1 \pm 2.2$ & $1.0 \pm 2.2$ & $t=-0.89, p=0.379$ \\
\hline BARS & $0.9 \pm 1.4$ & $0.6 \pm 1.1$ & $0.5 \pm 1.1$ & $t=-1.89, p=0.070$ \\
\hline Body weight (kg) & $67.6 \pm 12.4$ & $67.7 \pm 12.5$ & $67.8 \pm 12.6$ & $t=1.04, p=0.308$ \\
\hline BMI & $25.0 \pm 3.9$ & $25.0 \pm 4.1$ & $25.1 \pm 4.1$ & $t=1.61, p=0.118$ \\
\hline
\end{tabular}

Data are presented as mean \pm standard deviation.

PANSS, Positive And Negative Syndrome Scale; CDSS, Calgary Depression Scale for Schizophrenia; CGI-S, Clinical Global Impression Scale; CGI-CB, Clinical Global Impression Clinical Benefit Scale; SAS, Simpson Angus Scale; BARS, Barnes Akathisia Rating Scale; BMI, Body Mass Index. 
Table 4. Comparison of demographics, clinical scales, and treatment outcomes between the two starting dosage groups

\begin{tabular}{|c|c|c|c|}
\hline Variable & 200 mg start group $(n=10)$ & 400 mg start group $(n=19)$ & Comparison \\
\hline Age $(y r)$ & $48.1 \pm 13.5$ & $44.3 \pm 13.7$ & $t=0.70, p=0.485$ \\
\hline Sex, female & $3(30.0)$ & $11(57.9)$ & $\chi^{2}=2.04, p=0.153$ \\
\hline Duration of schizophrenia (yr) & $15.1 \pm 13.7$ & $14.2 \pm 8.7$ & $t=0.23, p=0.824$ \\
\hline Number of prior antipsychotics at baseline & $1.2 \pm 0.4$ & $1.2 \pm 0.4$ & $t=-0.06, p=0.949$ \\
\hline Average exposure dose ${ }^{a}$ of amisulpride (mg/day) & $230.0 \pm 48.3$ & $405.3 \pm 59.8$ & $t=-7.97, p<0.001$ \\
\hline BMI (baseline) & $24.5 \pm 4.8$ & $25.2 \pm 3.5$ & $t=-0.50, p=0.623$ \\
\hline BMI (change) ${ }^{b}$ & $0.2 \pm 0.4$ & $0.1 \pm 0.5$ & $t=0.07, p=0.945$ \\
\hline PANSS total (baseline) & $82.4 \pm 6.6$ & $81.4 \pm 14.9$ & $t=0.21, p=0.838$ \\
\hline PANSS total (change) ${ }^{b}$ & $-21.3 \pm 6.3$ & $-22.0 \pm 11.5$ & $t=-0.16, p=0.871$ \\
\hline CGI-S (baseline) & $4.4 \pm 0.7$ & $4.5 \pm 1.0$ & $t=-0.37, p=0.718$ \\
\hline CGI-S (change) ${ }^{b}$ & $-1.3 \pm 0.5$ & $-1.4 \pm 1.1$ & $t=0.19, p=0.849$ \\
\hline SAS (baseline) & $2.0 \pm 2.8$ & $0.9 \pm 2.4$ & $t=1.13, p=0.269$ \\
\hline SAS (change) ${ }^{b}$ & $0.0 \pm 0.0$ & $-0.4 \pm 1.8$ & $t=0.64, p=0.527$ \\
\hline BARS (baseline) & $0.7 \pm 1.3$ & $1.1 \pm 1.4$ & $t=-0.66, p=0.517$ \\
\hline BARS (change) ${ }^{b}$ & $-0.3 \pm 0.5$ & $-0.5 \pm 1.4$ & $t=0.37, p=0.714$ \\
\hline
\end{tabular}

Data are presented as mean \pm standard deviation or number (percentage).

BMI, Body Mass Index; PANSS, Positive And Negative Syndrome Scale; CGI-S, Clinical Global Impression Scale; SAS, Simpson Angus Scale; BARS, Barnes Akathisia Rating Scale.

${ }^{\mathrm{a}}$ Average daily dosage during 8 weeks. ${ }^{\mathrm{b}}$ Change is defined as subtraction of the baseline values from the values at week 8 .

$\chi^{2}$ : chi-squared test was used for statistical comparisons.

nificant differences between the two groups (PANSS total change: $t=-0.16, p=0.871$; CGI-S change: $t=0.19, p=$ 0.849) (Table 4).

\section{Safety Evaluation}

A total of 11 subjects did not complete the study; the reason for and time of withdrawal are shown in Table 2. The reasons for withdrawal were: lack of efficacy $(n=2$, $5 \%$ ), adverse reaction ( $n=3,7.5 \%$ ), lost to follow-up ( $n=$ $4,10 \%)$, and other reasons ( $n=2,5 \%)$. None of the subjects exhibited significant side effects severe enough to discontinue the medication and none had a causal relationship between drug administration and side effects.

There was no significant difference in $\operatorname{sex}\left(\chi^{2}=0.76, p=\right.$ $0.385)$, age $(t=-0.067, p=0.945)$, duration of illness $(t=$ $-0.903, p=0.372)$, number of prior antipsychotics $(t=$ $-0.173, p=0.864)$, duration of previous antipsychotics $(t=$ $0.814, p=0.421)$, starting dosage of amisulpride $(t=-0.109$, $p=0.914)$, and mean exposure dose of amisulpride $(t=$ $0.016, p=0.987)$ between completer and discontinuation groups.

There was no significant difference in the number of dropouts between the $200 \mathrm{mg}$ starting dose group and the $400 \mathrm{mg}$ starting dose group $\left(\chi^{2}=0.01, p=0.911\right.$; Table 2$)$. The severity of EPS based on SAS and BARS scores did not change significantly before and after 8 weeks of treatment
(Table 3). SAS decreased from $1.3 \pm 2.5$ (baseline) to $1.0 \pm$ 2.2 (at 8 weeks) $(t=-0.89, p=0.379)$ and BARS decreased from $0.9 \pm 1.4$ (baseline) to $0.5 \pm 1.1$ (at 8 weeks) $(t=-1.89, p=0.070)$. However, the change was not statistically significant (Table 3 ). The mean BMI changed from $25.0 \pm 3.9$ (baseline) to $25.1 \pm 4.1$ (at 8 weeks). The change was not statistically significant $(t=1.61, p=$ 0.118; Table 3). BMI, SAS scores, and BARS scores at baseline and changes in these scores after 8 weeks did not differ between the groups (BMI: $t=0.07, p=0.945$; SAS: $t=0.19, p=0.849$; BARS: $t=0.37, p=0.714$ ) (Table 4).

\section{DISCUSSION}

The main results of this study revealed that additional administration of amisulpride in patients with schizophrenia without response to SGAs was an effective treatment option, as $34.5 \%$ of the completers were responders (response of $50 \%$ or more). After amisulpride augmentation, PANSS domain scores and total PANSS scores showed significant improvement and side effects such as EPS and weight gain were not significant.

In this study, augmentation of previous SGAs with amisulpride in patients with schizophrenia lacking treatment response resulted in improvement of positive, negative, depressive, and general symptoms according to PANSS, 
CGI-S, CGI-CB, and CDSS scores after 8 weeks. A retrospective study of 15 patients with schizophrenia and schizoaffective disorder resistant to clozapine, olanzapine, risperidone, or ziprasidone reported that add-on therapy with amisulpride was useful in $80 \%$ of the patients [13].

An open observational study that used add-on therapy with amisulpride for 3 months in non-responders to risperidone monotherapy showed significant improvement in Brief Psychiatric Rating Scale, CGI-S, and Udvalg for Kliniske Undersøgelser Side Effect Rating Scale scores [11]. In patients who partially responded to olanzapine monotherapy, coadjuvant treatment with amisulpride for 3 months had a high response rate of $75.51 \%$ [12]. However, the authors admitted that the dosage of olanzapine was insufficient for some patients [12]. In an RCT involving administration of amisulpride or placebo in 68 patients with insufficient response to clozapine, amisulpride augmentation showed no significant improvement at 6 weeks, but there was greater chance of response and improvement in negative symptoms at the 12-week follow-up [14]. However, amisulpride was associated with a greater side effect burden including cardiac side effects [14]. Previous studies on this topic have shown that in a more well-designed study such as an RCT, the usefulness of augmentation was less pronounced and the response improved with longer treatment periods when compared with an open study.

Previously, antipsychotic monotherapy was recommended in pharmacotherapy guidelines for schizophrenia and polypharmacy was generally avoided if possible [23]. However, a recent study on the association among antipsychotic monotherapy, polypharmacy type, and rehospitalization in patients with schizophrenia revealed that combining aripiprazole with clozapine was associated with the lowest risk of rehospitalization. The authors insisted that correction of treatment guideline is needed in the future [24]. The current revision of the American Psychiatric Association guideline states that augmentation with another antipsychotic can be considered, but recommends that the trial of clozapine should not be delayed due to the augmentation process, since there is established evidence for the better effect of clozapine on treatment resistant schizophrenia [25].

In this study, there was no deterioration of EPS after addition of amisulpride and no change in BMI of the parti- cipants. Although there was no statistically significant change, BARS scores showed a trend toward decrease at 8 weeks when compared with baseline $(t=-1.89, p=$ 0.070 ). In a previous study regarding augmentation of oral or parenteral risperidone with amisulpride, EPS had generally improved at the last visit after the augmentation with amisulpride [11]. Another study regarding augmentation of olanzapine with amisulpride also showed significant decrease in the number of patients suffering from moderate EPS at 12 weeks after treatment [12]. However, an RCT that compared the augmentation of clozapine with amisulpride and placebo showed that the amisulpride augmentation group revealed a greater number of side effects such as weight gain, sexual dysfunction, and aversive subjective experiences according to the Antipsychotic Non-Neurological Side Effects Scale assessments, which was designed to systematically and comprehensively assess the full range of side effects [14].

In the present study, there was no significant difference in the treatment response and side effects between the $200 \mathrm{mg}$ starting dose group and the $400 \mathrm{mg}$ starting dose group (Table 4 ). There were no significant differences between the two dosage groups in terms of change in $\mathrm{BMI}$ as well as in terms of changes in PANSS, CGI-S, SAS, and BARS scores. However, the number of subjects in the 200 $\mathrm{mg}$ and the $400 \mathrm{mg}$ groups was rather small and further studies are needed in the future. In a 6-week randomized open-label study of amisulpride in acute exacerbation of schizophrenia, no statistically significant differences were observed in the overall incidence of adverse events between the $400 \mathrm{mg}$ and the $800 \mathrm{mg}$ dose groups [26]. In order to confirm and verify the results of the present study, an RCT comparing various doses of amisulpride and placebo will be needed in the future.

Our study has several limitations. Due to the nature of this being an open-label, prospective study, this study did not have a control group (patients taking SGAs without add-on therapy). The small sample size also makes it difficult to generalize our results. In addition, the possibility that the improvement of symptoms after administration of amisulpride was due to the previous antipsychotics cannot be excluded [27].

In conclusion, the present study suggested that augmentation with amisulpride was effective and safe in patients with schizophrenia who lacked treatment response to SGAs. In clinical practice, this option is expected to be 
an effective and safe treatment strategy. Future studies including a greater number of subjects and placebo groups are needed to validate the results of the present study.

\section{Acknowledgments}

Sponsorship for this study was funded by the Handok Inc., Seoul, Republic of Korea. The funders had no role in study design, data collection and analysis, decision to publish, or preparation of the manuscript.

\section{Conflicts of Interest}

No potential conflict of interest relevant to this article was reported.

\section{Author Contributions}

Conceptualization: Seong-Jin Cho. Data acquisition: Seung-Gul Kang, Seo-Eun Cho, Kyoung-Sae $\mathrm{Na}$, and Seong-Jin Cho. Formal analysis: Seung-Gul Kang and Kyoung-Sae Na. Funding: Seong-Jin Cho. Supervision: Seong-Jin Cho and Chi-Un Pae. Writing-original draft: Seung-Gul Kang. Writing - review \& editing: Seong-Jin Cho.

\section{ORCID}

Seung-Gul Kang

https://orcid.org/0000-0003-4933-0433

Seo-Eun Cho https://orcid.org/0000-0002-3991-2192

Kyoung-Sae Na https://orcid.org/0000-0002-0148-9827

Chi-Un Pae https://orcid.org/0000-0003-1632-4248 Seong-Jin Cho https://orcid.org/0000-0002-8814-5807

\section{REFERENCES}

1. Menezes NM, Arenovich T, Zipursky RB. A systematic review of longitudinal outcome studies of first-episode psychosis. Psychol Med 2006;36:1349-1362.

2. Lally J, MacCabe JH. Antipsychotic medication in schizophrenia: a review. Br Med Bull 2015;114:169-179.

3. Srisurapanont M, Suttajit S, Maneeton N, Maneeton B. Efficacy and safety of aripiprazole augmentation of clozapine in schizophrenia: a systematic review and meta-analysis of randomized-controlled trials. J Psychiatr Res 2015;62:38-47.

4. Paton C, Whittington C, Barnes TR. Augmentation with a second antipsychotic in patients with schizophrenia who partially respond to clozapine: a meta-analysis. J Clin Psychopharmacol 2007;27:198-204.

5. Hatta K, Hasegawa H, Imai A, Sudo Y, Morikawa F, Katayama $\mathrm{S}$, et al. Real-world effectiveness of antipsychotic monotherapy and polytherapy in 1543 patients with acute-phase schizophrenia. Asian J Psychiat 2019;40:82-87.
6. Galling B, Roldán A, Hagi K, Rietschel L, Walyzada F, Zheng $\mathrm{W}$, et al. Antipsychotic augmentation vs. monotherapy in schizophrenia: systematic review, meta-analysis and metaregression analysis. World Psychiatry 2017;16:77-89.

7. Puech A, Fleurot O, Rein W. Amisulpride, and atypical antipsychotic, in the treatment of acute episodes of schizophrenia: a dose-ranging study vs. haloperidol. The Amisulpride Study Group. Acta Psychiatr Scand 1998;98:65-72.

8. Möller HJ, Boyer P, Fleurot O, Rein W. Improvement of acute exacerbations of schizophrenia with amisulpride: a comparison with haloperidol. PROD-ASLP Study Group. Psychopharmacology (Berl) 1997;132:396-401.

9. Mortimer A, Martin S, Lôo H, Peuskens J; SOLIANOL Sudy Group. A double-blind, randomized comparative trial of amisulpride versus olanzapine for 6 months in the treatment of schizophrenia. Int Clin Psychopharmacol 2004;19:63-69.

10. Leucht S, Cipriani A, Spineli L, Mavridis D, Orey D, Richter F, et al. Comparative efficacy and tolerability of 15 antipsychotic drugs in schizophrenia: a multiple-treatments meta-analysis. Lancet 2013;382:951-962.

11. Toledo-Romero F, Molina JD, López-Rodríguez E, Amorin-Díaz M, Muñoz Algar MJ, Aparicio-Castro E. Augmentation with amisulpride for schizophrenic patients non-responsive to risperidone monotherapy. Pharmacopsychiatry 2015;48:51-57.

12. Molina JD, Toledo-Romero F, López-Rodríguez E, Amorin-Díaz M, Lerma-Carrillo I, López-Muñoz F. Augmentation treatment with amisulpride in schizophrenic patients partially responsive to olanzapine. Pharmacopsychiatry 2011;44:142-147.

13. Lerner V, Bergman J, Borokhov A, Loewenthal U, Miodownik C. Augmentation with amisulpride for schizophrenic patients nonresponsive to antipsychotic monotherapy. Clin Neuropharmacol 2005;28:66-71.

14. Barnes TR, Leeson VC, Paton C, Marston L, Davies L, Whittaker W, et al. Amisulpride augmentation in clozapine-unresponsive schizophrenia (AMICUS): a double-blind, placebo-controlled, randomised trial of clinical effectiveness and cost-effectiveness. Health Technol Assess 2017;21:1-56.

15. American Psychiatric Association. Diagnostic and statistical manual of mental disorders (DSM- $\left.5^{\mathbb{R}}\right)$. Arlington:American Psychiatric Publishing;2013.

16. Kay SR, Fiszbein A, Opler LA. The positive and negative syndrome scale (PANSS) for schizophrenia. Schizophr Bull 1987; 13:261-276.

17. Guy W. ECDEU assessment manual for psychopharmacology. Rockville: U.S. Dept. of Health, Education, and Welfare, Public Health Service, Alcohol, Drug Abuse, and Mental Health Administration, National Institute of Mental Health, Psychopharmacology Research Branch, Division of Extramural Research Programs; 1976.

18. Kim YK, Won SD, Lee KM, Choi HS, Jang HS, Lee BH, et al. A study on the reliability and validity of the Korean Version of the Calgary Depression Scale for Schizophrenia (K-CDSS). J Korean Neuropsychiatr Assoc 2005;44:446-455. 
19. Addington D, Addington J, Maticka-Tyndale E. Assessing depression in schizophrenia: the Calgary Depression Scale. Br J Psychiatry Suppl 1993;(22):39-44.

20. Barnes TR. A rating scale for drug-induced akathisia. Br J Psychiatry 1989;154:672-676.

21. Simpson GM, Angus JW. A rating scale for extrapyramidal side effects. Acta Psychiatr Scand Suppl 1970;45(S212):11-19.

22. Leucht S, Davis JM, Engel RR, Kissling W, Kane JM. Definitions of response and remission in schizophrenia: recommendations for their use and their presentation. Acta Psychiatr Scand Suppl 2009;(438):7-14.

23. Lehman AF, Lieberman JA, Dixon LB, McGlashan TH, Miller $\mathrm{AL}$, Perkins DO, et al. Practice guideline for the treatment of patients with schizophrenia. Am J Psychiatry 2004;161(2 Suppl):1-56.

24. Tiihonen J, Taipale H, Mehtälä J, Vattulainen P, Correll CU, Tanskanen A. Association of antipsychotic polypharmacy vs monotherapy with psychiatric rehospitalization among adults with schizophrenia. JAMA Psychiatry 2019;76:499-507.

25. American Psychiatric Association. Practice guideline for the treatment of patients with schizophrenia [Internet]. American Psychiatric Association [cited at 2020 May 12]. Available from: https://www.psychiatry.org/psychiatrists/practice/clinical-practice-guidelines/guideline-development-process.

26. Lee SJ, Lee JH, Jung SW, Koo BH, Choi TY, Lee KH. A 6-week, randomized, multicentre, open-label study comparing efficacy and tolerability of amisulpride at a starting dose of 400 $\mathrm{mg} /$ day versus $800 \mathrm{mg} /$ day in patients with acute exacerbations of schizophrenia. Clin Drug Investig 2012;32:735-745.

27. Kahn RS, Winter van Rossum I, Leucht S, McGuire P, Lewis SW, Leboyer M, et al. Amisulpride and olanzapine followed by open-label treatment with clozapine in first-episode schizophrenia and schizophreniform disorder (OPTiMiSE): a three-phase switching study. Lancet Psychiatry 2018;5:797-807. 